\title{
Thermal imaging in the 3-5 micron range for precise localization of defects: application on frescoes at the Sforza Castle
}

\author{
Claudia Daffara ${ }^{\mathrm{a}}$, Simone Parisotto ${ }^{\mathrm{b}}$, Sara Mazzocato ${ }^{\mathrm{a}}$, Paola Ilaria Mariottic ${ }^{\mathrm{c}}$, and Dario \\ Ambrosini $^{\mathrm{d}}$
}

aDept. of Computer Science, University of Verona, st. le Grazie 15, I-37134, Verona, Italy

b Dept. of Applied Mathematics and Theoretical Physics, University of Cambridge, Wilberforce Road, Cambridge CB3 0WA, United Kingdom

${ }^{\mathrm{c}}$ Opificio delle Pietre Dure, v.le F. Strozzi, 1 (Fortezza da Basso), I-50129, Firenze, Italy

${ }^{\mathrm{d}}$ Dept. of Industrial and Information Engineering and Economics (DIIIE), University of

L'Aquila, pl. Pontieri 1, I-67100, L'Aquila, Italy

\begin{abstract}
Infrared methods are of great importance in nondestructive testing of artworks, allowing a remote and wide-field imaging of interesting hidden features. Here we discuss a workflow based on thermal imaging in the mid infrared 3-5 micron range for the evaluation of subsurface defects in frescoes. Particular attention is payed to obtaining a high resolution (submillimetric) localization of the defects. The transfer of diagnostics techniques into real world applications, is discussed through the proof of concept of the proposed workflow on frescoes at the Sforza Castle (Milan, Italy).
\end{abstract}

Keywords: Mid infrared, frescoes, detachments, defect detection, thermal quasi-reflectography, non-invasive analysis, thermal imaging

\section{INTRODUCTION}

Infrared methods are important tools in nondestructive testing of paintings and frescoes, allowing a remote and wide-field imaging of interesting hidden features. Simply said, the methods based on the thermal emissivity response of the target surface, i.e. thermography in the LWIR and MWIR bands, ${ }^{1,2}$ are being used for probing the structural defects (also deep), while, the methods based on the reflectance response of the materials, i.e. reflectography ${ }^{3-5}$ in the NIR and SWIR bands, provide clearer information about the distribution of pictorial matter in the layered, subtle, paintings (pigments, morphological "artistic" features).

To the second class of methods, we must add the Thermal Quasi-Reflectography ${ }^{6,7}$ (TQR) technique (a kind of reflectography technique in the mid-infrared range in which the thermal spurious contribution is controlled and minimized), proposed by the same authors that is well accepted by the community ${ }^{8,9}$ for the results obtained on the frescoes, and adopted for the recent restoration of two Italian masterpieces: The Resurrection by Piero della Francesca and the Sala delle Asse frescoed by Leonardo da Vinci. ${ }^{10}$

Thermography in the LWIR band from $8 \mu \mathrm{m}$ to $12 \mu \mathrm{m}$, usually suitable for investigating the painting support, does not allow a sharp imaging, which is possible in principle, but difficult in practice. Therefore it is not able to solve effectively a crucial problem of the field, namely, the investigation of those structural shallow defects that require a fine, high (spatial) resolved mapping: an exemplar problem is the location of the small detached areas of pictorial paint and plaster layers in frescoes. Another problem not solved yet in thermal imaging is that, in order to obtain an effective mapping, image mosaicking and spatial registering of thermograms to visible images are required, but the absence of reference points make these tasks difficult.

Further author information: (Send correspondence to C.D.)

C.D.: E-mail: claudia.daffara@univr.it 
Conversely, MWIR research-grade cameras from $3 \mu \mathrm{m}$ to $5 \mu \mathrm{m}$, also used in artworks thermography, allow to perform a novel imaging in dual-mode, in which thermography and quasi-reflectography are integrated in a two-step measurement workflow. The two thermal datasets (reflective and emissive mode) are then jointly analyzed to map both surface and subsurface features in painting. ${ }^{7}$

In this paper we discuss a detailed application of the dual-mode imaging technique to the diagnostics of frescoes, with an emphasis on the location of detachments. We elaborate on the above mentioned problem of registering spatially the thermal images to the reference visible, thus obtaining fine resolution mapping through thermal mosaicking. The proposed workflow is modular and allows for processing the thermal sequence in time with the thermographic techniques available in literature.

As proof of concept, we present the last results on the application of dual-mode imaging on the frescoes by Leonardo da Vinci located in the Sforza Castle (Milan, Italy). After a restoration lasting several years, the masterpieces were recently presented to the public. The dual-mode technique was used to guide the conservators during the restoration works, thus, by showing an effective way to investigate cultural heritage, this research could bring innovation in artwork diagnostics.

\section{BASIC PRINCIPLES}

The dual-mode imaging method ${ }^{7}$ is based on the acquisition of two radiometric datasets with the same thermal camera: the TQR measurement in reflection mode and the sequence of thermograms in emission mode. The geometry and the optical configuration of the set up was fixed and this allowed to obtain spatially registered measurements.

The TQR imaging technique is based on the observation that at room temperature, e.g. $293 \mathrm{~K}$, an object emits approximately $1 \%$ of its thermal energy in the range from $3 \mathrm{um}$ to $5 \mathrm{um}$. Therefore, if MWIR radiation is send to the painting, the acquired signal is dominated by the reflected radiation that strongly depends on surface structure. Thus the technique is very powerful in detecting different surface materials such as the original ones as well as materials from restoration and deterioration.

Moreover, from the TQR signal is possible to estimate the point-wise emissivity of the surface, assuming the grey body approximation as valid in this case and considering that MWIR wavelenghts do not penetrate the painting layers.

The second spatially registered radiometric dataset is composed by the "traditional" thermal sequence recorded by the camera in emission modality.

\subsection{Processing}

The processing of the dual mode imaging is exemplified in Figure 1.

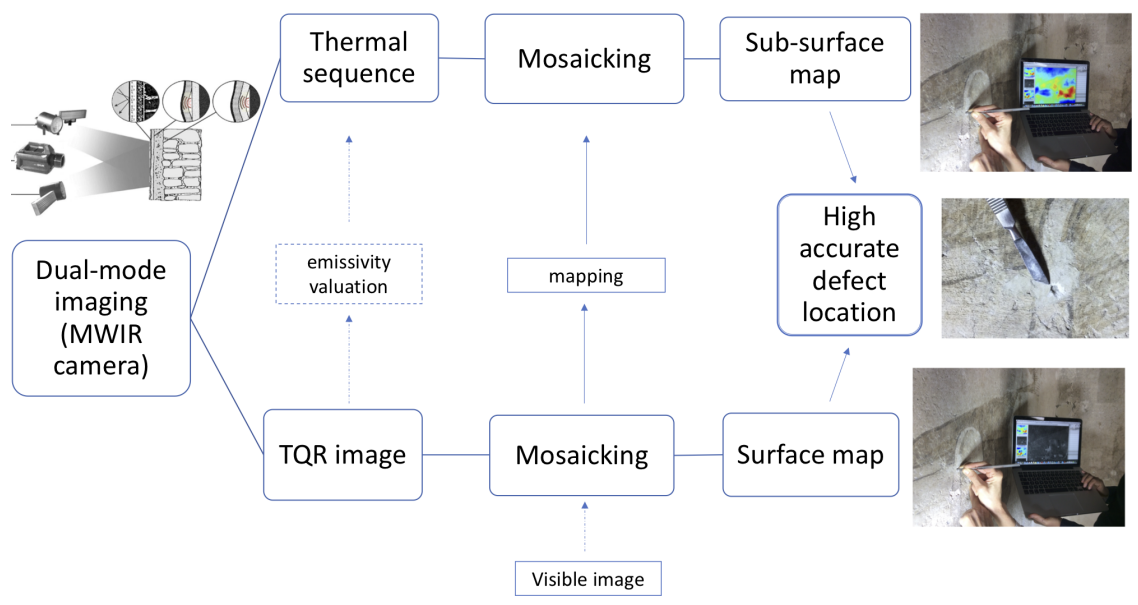

Figure 1. Measurement and processing workflow for accurate defect mapping. 
The radiometric TQR images were recorded using a custom designed non heating source and subsequently calibrated using a certified reflectance standard. The reflectance map is obtained from each image $I$ taking as dark image the thermal emission $I^{\mathrm{b}}\left(T_{\mathrm{obj}}\right)$, as follows

$$
R_{i j}=R_{\mathrm{ref}} \frac{I_{i j}-I_{i j}^{\mathrm{b}}\left(T_{\mathrm{obj}}\right)}{I_{\mathrm{ref}}-I_{i j}^{\mathrm{b}}\left(T_{\mathrm{obj}}\right)},
$$

where $I_{\text {ref }}$ and $R_{\text {ref }}$ are the TQR radiometric image and the nominal reflectance of the reference white.

The thermal sequence is analyzed starting from the observation that the heat wave propagation in the "regular" non-defective area and in defective area is different. In detail, the defect induces an alteration of the surface temperature field that is visible in the recorded image.

In the regular non defective region the components due to the later diffusion vanish and the heat diffusion is approximated by the $1 \mathrm{D}$ equation in (2):

$$
\frac{\partial T}{\partial t}=\alpha \frac{\partial^{2} T}{\partial z^{2}}
$$

Launching a Dirac pulse stimulus, the deviation of trend of $\Delta T$ from the well-known power law $\left(\Delta T(t) \propto t^{-1 / 2}\right)$ implies the presence of a bulk discontinuity.

The detachments trap air and hence they are seen as resistive defects with high thermal resistance. Similarly, one can try to detect thermal conductive discontinuities due to restoration materials previously applied. Visually, they can be seen as "hot" and "cold" defects in the thermograms.

As stated before, the main drawback of the thermal measurement is the blurring that prevents from the accurate location of the defects. The power of the proposed method lies in referencing and mosaicking the TQR signal in the visible space and consequently mapping the thermal image thanks to the coupling of the two thermal datasets.

\section{EXPERIMENTAL RESULTS}

\subsection{Detecting the defects}

Simply said, defects are discontinuities that change the heat propagation across the painting matter. We can model the fresco as a layered structure, with the heat flow in the orthogonal direction and neglecting the lateral diffusion. However, there are highly non homogeneous materials that lead to the presence of both resistive and conductive defects, e.g. unrestored and restored plaster detachments. The situation is of course very far from that of non destructive techniques carried out in a controlled tested environment. In the raw thermographic signal, the alterations may be detected from the overall image contrast with the background signal during the cooling, namely, as regions in which some anomalous behaviour of the surface temperature is measured. Enhancement techniques based on definitions of a contrast between the tested area and a reference one improve the detection, but the identification of suitable "nondefective" region is difficult in artworks. Therefore, coming to the realworld application of ancient artistic manufacts, the condition to have thermal images precisely referenced to the surface is mandatory, also allowing the supervision of the restorer.

The Figure 2 below shows an example of the result obtainable from the dual mode dataset: the thermal sequence can be visually explored in an improved way, thanks to the spatially aligned TQR images, which allow the localization of the features in the fresco. If some calculation on thermal data is carried out, mixing pixel wise the image planes at different times, the result of the processing inherits the referentiation. This is the case of the Standard Contrast reported in the Figure below. 

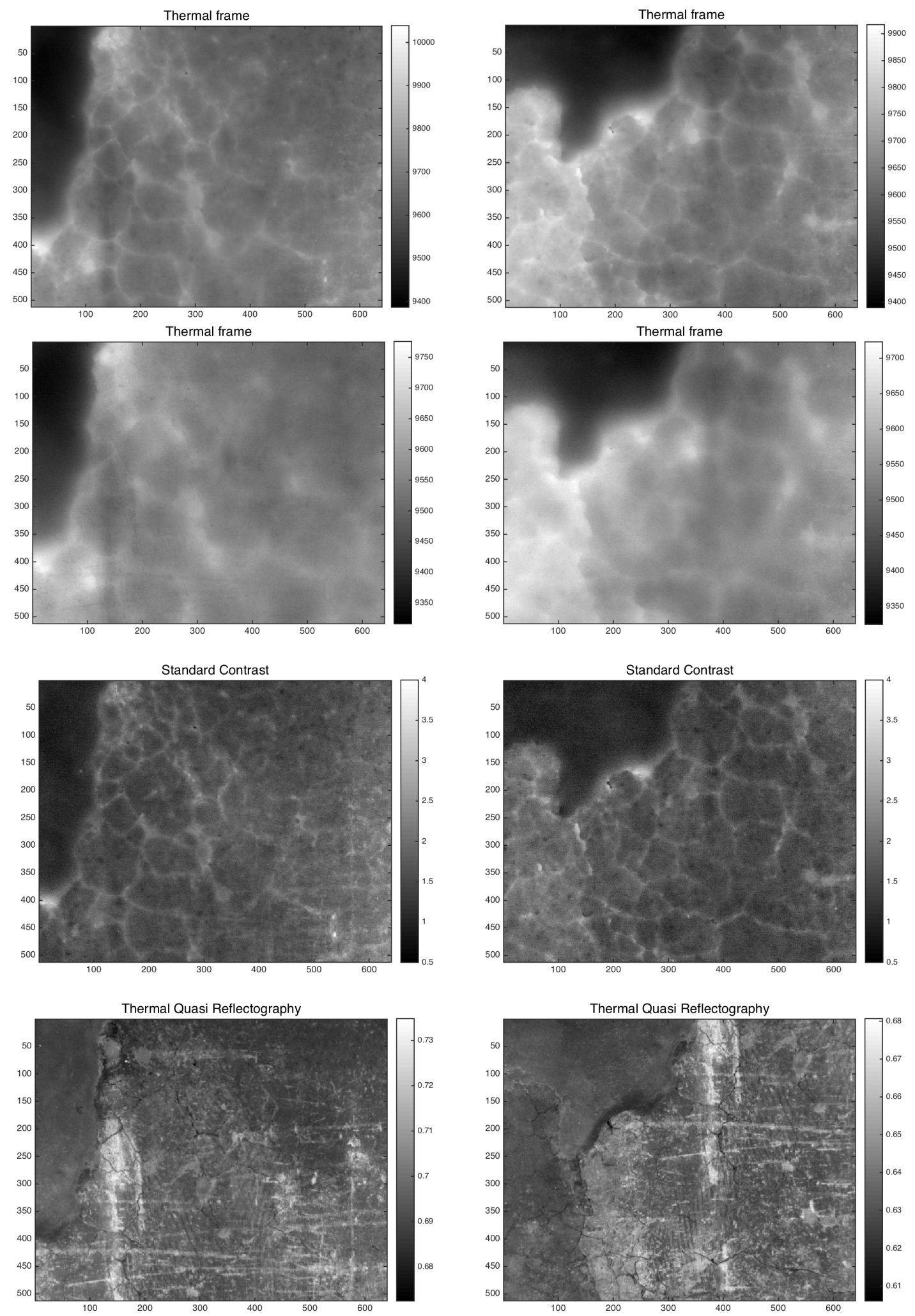

Figure 2. Thermal frames at the beginning $(1 \mathrm{~s})$ and during $(10 \mathrm{~s})$ the cooling (first and second row), Contrast map (third row). The thermal images, as well as the calculated contrast are referenced to the surface through the Mid Infrared (quasi) reflectogram (last row). 


\subsection{Exploiting the dual model dataset for mosaicking referenced maps}

The thermal dataset on the frescoes of the Sala delle Asse has been acquired at a remarkable high resolution, which is not typical in the practice of thermography applied to large areas. The pixel at object plane is less than $0.5 \mathrm{~mm}$. A huge number of frames of $512 \times 640$ pixels were taken to scan the wall hosting the mural painting attributable to Leonardo, with the contiguous pairs of images overlapped of the necessary portion for carrying out an accurate mosaicking.

The Figure 3 reports as result an example of how the dual mode methods enabled the thermal mosaicking process, a difficult task that cannot be performed alone, as early mentioned, due to the difficulty in finding "visible" reference points in the relaxing phase thermograms with the required accuracy. Following the workflow depicted in Figure 1, by mosaicking firstly the reflectance TQR images, which contains lot of features down to the diffraction-limit, the obtained transformation can be applied secondly to the thermography dataset, which is aligned to the TQR dataset, thus obtaining a mosaic also in the thermal range. Figure 3 have been composed using the cracks and other visible traces (as the salts decay) that are visible in the fresco surface and orthophoto, which was at disposal during the restoration of the frescoes and used as plane to reference the various analysis. As reported in literature, quasi reflectography in the MWIR range is a technique highly sensitive to surface materials. ${ }^{6}$ Beside the mosaicking, it was possible to reference the dual mode dataset to the visible surface using the orthophoto.
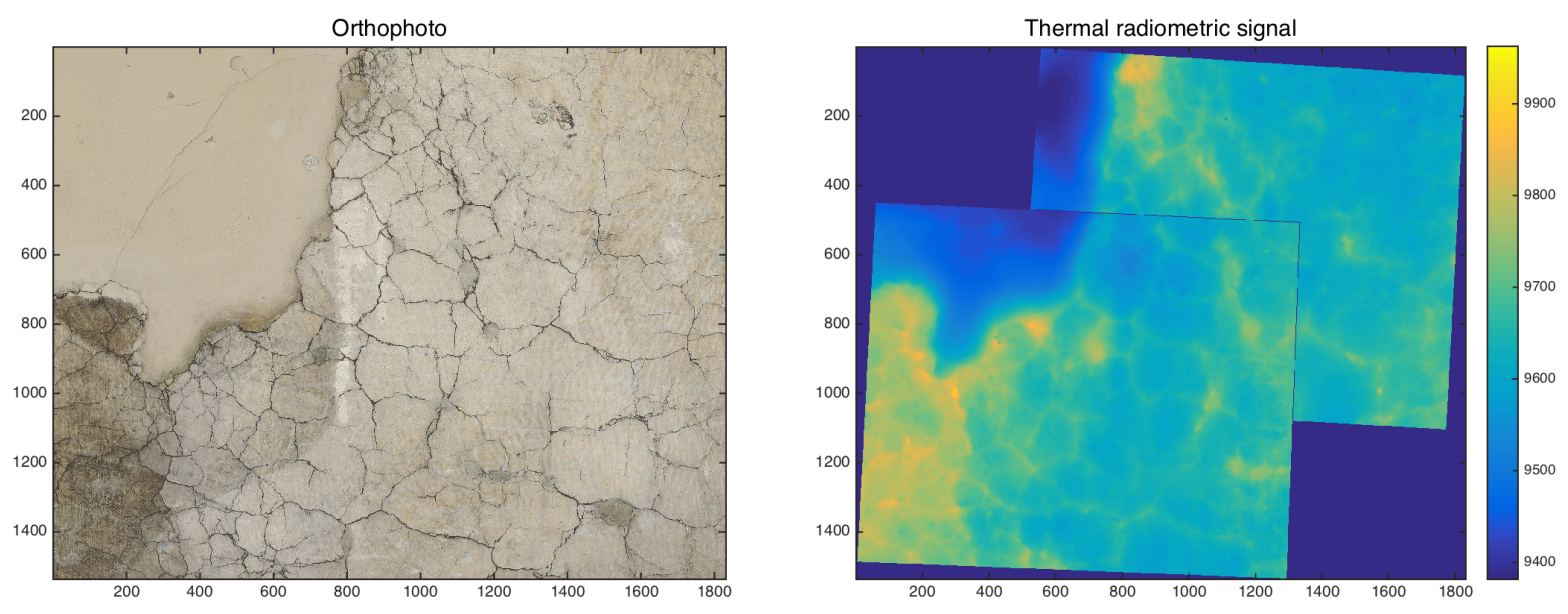

Figure 3. Thermal mosaic spatially registered to an orthophoto reference map (detail with two frames extracted).

\subsection{Advanced processing on the referenced thermographic sequence towards a quantitative defects analysis}

The last example treats the temporal thermographic signal as a whole. The Figure 4 depicts the behaviour in time of the thermographic signal in the cooling phase for a spot in an anomalous "defective" and in a "regular" region of the surface of the fresco.

There are a number of techniques that are used to model the signal $T(t)$ on analytical basis, starting from the heat transfer theory and the choice of assumptions regarding the system property. The most simple model is the ideal-slab that treats the propagation of the heat wave in the depth direction, neglecting the lateral components and solving the 1D dimensional Fourier equation (2). This can be extended to consider also lateral diffusion and other effects.

Following the ideal model of the long pulse decay in time shown in Figure 4, we conveniently approximate the signal by a sum of polynomial terms in the log-log space. ${ }^{11}$ As this process is done point wisely, for each point in the matrix independently, the referentiation mechanism of the dual mode technique is still valid. 

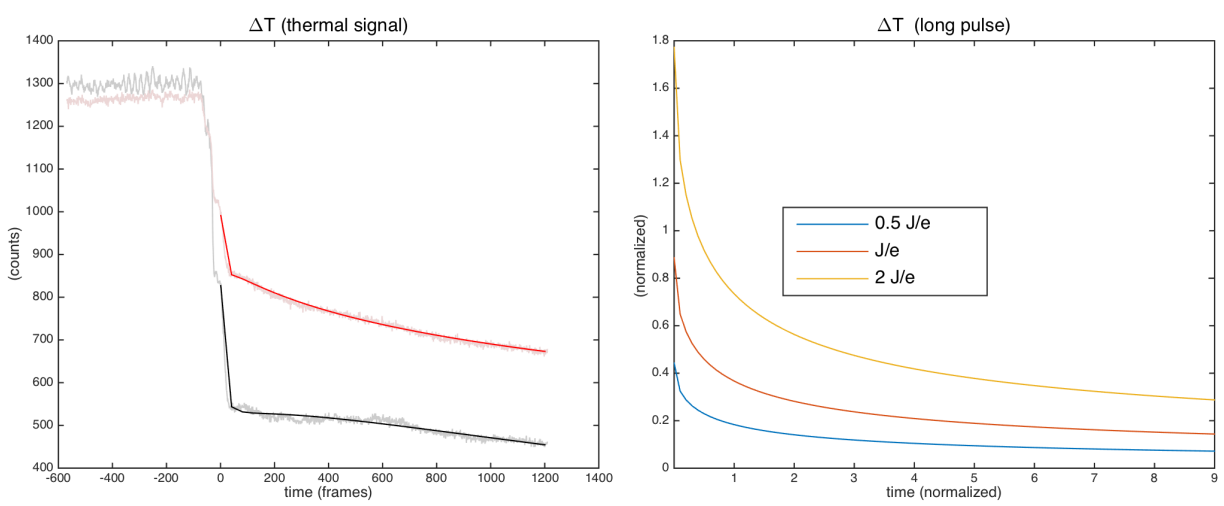

Figure 4. Left: thermal signal sampled by the camera in a single pixel $(<0.5 \mathrm{~mm}$ at object plane $)$ at $120 \mathrm{~Hz}$ and the interpolated curve for further analysis. Two spots with different behaviour are shown. Right: long pulse theoretical model for the ideal-indefinite-slab for two different mediums ( $J$ is the heat pulse and $e$ the thermal effusivity).

In the Figure 5 below it is shown the separation of the thermal signal during the cooling phase: the first term contains the mean signal, it is the dominant part of the thermogram, showing the response of the object to the heating absorption; in the second term the network of detaches is more detectable; the last term contains the weak residual signal. Both the maps are spatially superimposed to the TQR image and to the visible high resolution ortophoto, which together allow the user a more comprehensive analysis of the thermal data and interpretation of the defects.

Following the proposed workflow, we report in Figure 6 the mosaicking of the dominant first term and the second term of the signal.
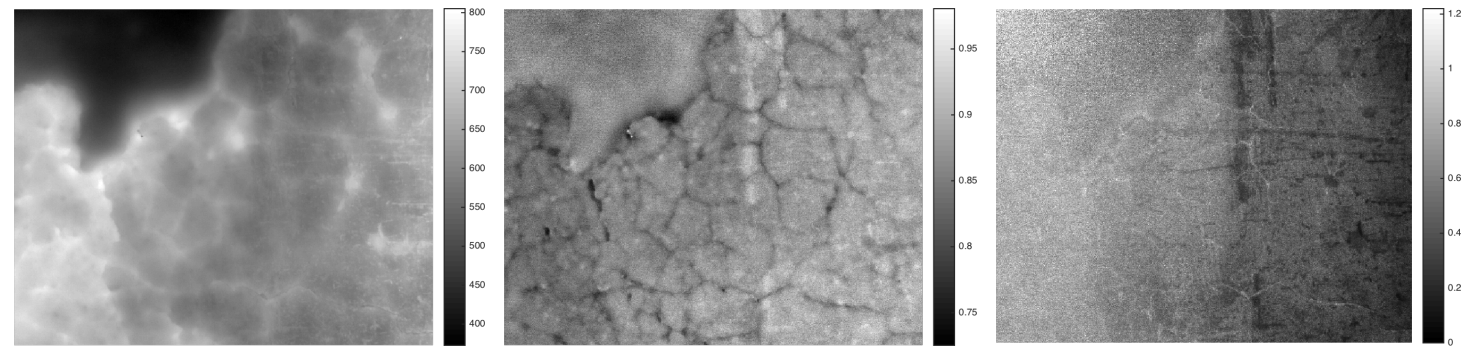

Figure 5. Decomposition of the thermal signal during the cooling phase $(t=10 \mathrm{~s})$. From left to right: first term, second term, residual term.
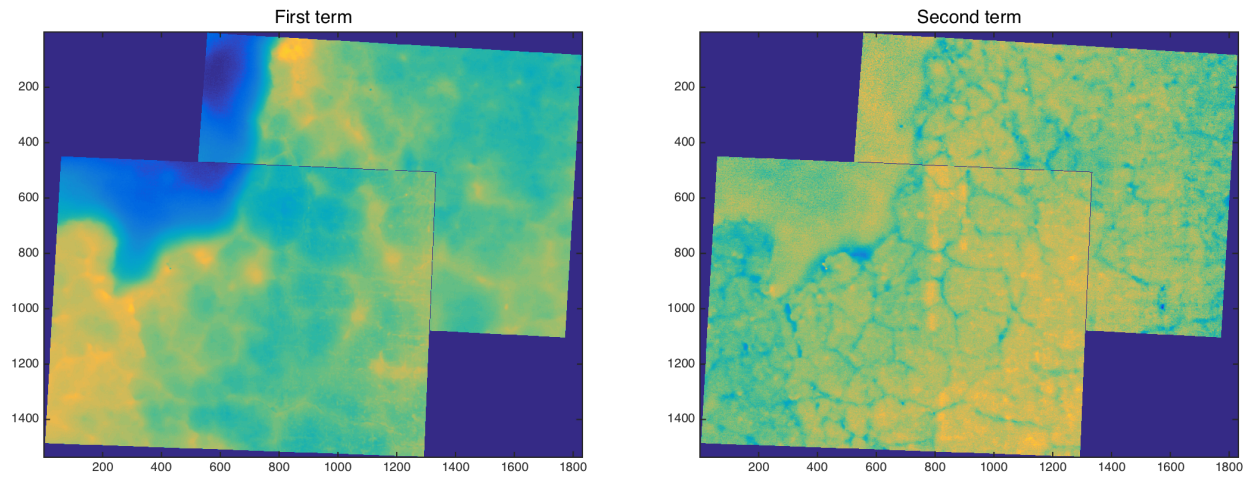

Figure 6. Mosaic processing after signal decomposition. 


\section{CONCLUSIONS: GETTING INTO REAL-WORLD APPLICATIONS}

Unlike in the case of industrial applications, the presence of extremely heterogeneous and unknown materials is a challenge when applying thermal imaging to artworks. Moreover, working in the real scenario where you can not control all the variables such as vibrations, is very different from working in a laboratory. Notwithstanding, this last section shows the power of technique during the restoration operations. During the restoration, the areas of measurement were conceived with intensive interdisciplinary work and continuous interaction between restorers and scientists. An expert restorer recognizes detached and "safe" areas manually "knocking" on the wall and discriminating the different sound response, but a manual inspection cannot produce an accurate map of the defects localization.

Figure 7 illustrates an example of the setup, the investigated area and the results. In particular, the bottom row shows the thermogram, the TQR and their overlap respectively. As reported above, the thermograms allow to bring out the discontinuities in the materials and the intrinsic problem of localization is overcome because they are fully spatially aligned with the TQR which in turn is referenced to the visible image allowing an accurate one-to-one map of the detachments. The integration of the TQR and thermal datasets allows, for instance, to study the plaster detachments distinguishing the material used to construct the sub-layer of the stucco work: for example, cement characterised by less thermal resistance or mortar.

Summing up, the proposed approach is built upon the key features of both thermography and TQR (the ability to detect sub-superficial defects and the capability to differentiate surface materials with a high spatial resolution, respectively). Recognizing the complementary of these features, and using them in a synergic way, led to a diagnostic tool that exhibits thermographic defects detection coupled with unprecedented resolution in spatial localization. Furthermore, it was proved capable of working in a hostile environment such as a restoration yard.
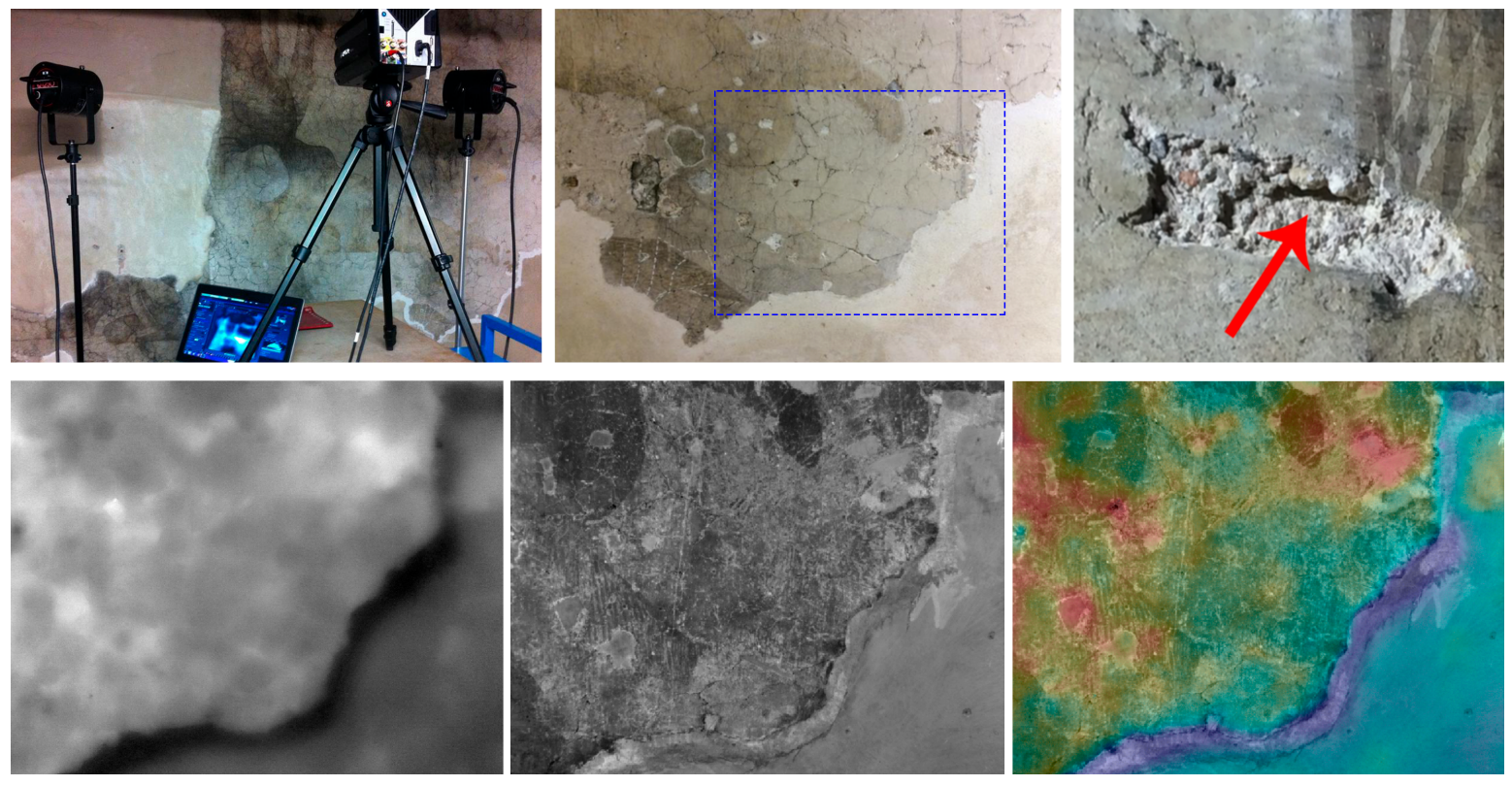

Figure 7. Top row: the setup in the field, an example of the analyzed area and an example of defect. Bottom row: thermogram, TQR and their overlap of the blue area highlighted above.

\section{ACKNOWLEDGMENTS}

SP acknowledges the support from the Leverhulme Trust Research Project Grant (RPG-2018-121) "Unveiling the Invisible - Mathematics for Conservation in Arts and Humanities". We thank Dr. Francesca Tasso (Soprintendenza of Castello Sforzesco, Milan, Italy) for supporting the on-field intervention on Leonardo frescoes. 


\section{REFERENCES}

[1] Maldague, X., [Theory and practice of infrared technology for nondestructive testing], Wiley, New York (2001).

[2] Gavrilov, D., Maev, R. G., and Almond, D., "A review of imaging methods in analysis of works of art: Thermographic imaging method in art analysis," Canadian Journal of Physics 92(4), 341-364 (2014).

[3] De Boer, J. V. A., "Infrared reflectography: a method for the examination of paintings," Applied Optics 7(9), 1711-1714 (1968).

[4] Delaney, J. K., Zeibel, J. G., Thoury, M., Littleton, R., Palmer, M., Morales, K. M., de La Rie, E. R., and Hoenigswald, A., "Visible and infrared imaging spectroscopy of picasso's harlequin musician: mapping and identification of artist materials in situ," Applied spectroscopy 64(6), 584-594 (2010).

[5] Daffara, C. and Fontana, R., "Multispectral infrared reflectography to differentiate features in paintings," Microscopy and Microanalysis 17(5), 691 (2011).

[6] Daffara, C., Ambrosini, D., Pezzati, L., and Paoletti, D., "Thermal quasi-reflectography: a new imaging tool in art conservation," Optics Express 20(13), 14746-14753 (2012).

[7] Daffara, C., Parisotto, S., and Ambrosini, D., "Multipurpose, dual-mode imaging in the 3-5 $\mu \mathrm{m}$ range (mwir) for artwork diagnostics: A systematic approach," Optics and Lasers in Engineering 104, 266-273 (2018).

[8] Borg, B., Dunn, M., Ang, A., and Villis, C., "The application of state-of-the-art technologies to support artwork conservation: Literature review," Journal of Cultural Heritage 44, 239-259 (2020).

[9] Orazi, N., "Mid-wave infrared reflectography and thermography for the study of ancient books: A review," Studies in Conservation 65(8), 437-449 (2020).

[10] Daffara, C., Parisotto, S., and Mariotti, P. I., "Mid-infrared thermal imaging for an effective mapping of surface materials and sub-surface detachments in mural paintings: integration of thermography and thermal quasi-reflectography," Proc. SPIE 9527, 91-102 (2015).

[11] Shepard, S. M., Lhota, J. R., Rubadeux, B. A., Wang, D., and Ahmed, T., "Reconstruction and enhancement of active thermographic image sequences," Optical Engineering 42(5), 1337-1342 (2003). 